\title{
Prospective feasibility study for single-tracer sentinel node mapping by ICG (indocyanine green) fluorescence and OSNA (one-step nucleic acid amplification) assay in laparoscopic gastric cancer surgery
}

\author{
Yoshiaki Shoji ${ }^{1}$ Koshi Kumagai ${ }^{1}$ - Satoshi Kamiya ${ }^{1}$ - Satoshi Ida ${ }^{1}$. Souya Nunobe ${ }^{1}$. Manabu Ohashi ${ }^{1}$. \\ Shoichi Yoshimizu² ${ }^{2}$ Yusuke Horiuchi ${ }^{2}$. Toshiyuki Yoshio ${ }^{2}$ - Akiyoshi Ishiyama ${ }^{2}$. Toshiaki Hirasawa ${ }^{2}$. Tomo Osako ${ }^{3}$. \\ Noriko Yamamoto ${ }^{3} \cdot$ Junko Fujisaki $^{2} \cdot$ Takeshi Sano $^{1} \cdot$ Naoki Hiki $^{1}$ (D)
}

Received: 7 September 2018 / Accepted: 22 December 2018 / Published online: 3 January 2019

(c) The International Gastric Cancer Association and The Japanese Gastric Cancer Association 2019

\begin{abstract}
Background The double-tracer method has been established for sentinel node (SN) mapping in gastric cancer surgery. However, there remain several unresolved issues that prevent its widespread use in clinical practice. In this study, we aimed to demonstrate the feasibility of single-tracer SN mapping in laparoscopic surgery for gastric cancer, using indocyanine green (ICG) fluorescence imaging with a one-step nucleic acid amplification (OSNA) assay intraoperatively.

Methods Patients with clinical T1N0M0 gastric adenocarcinoma preoperatively were considered for inclusion if they had a single primary lesion $4 \mathrm{~cm}$ or less in maximal diameter. Immunohistochemical staining with the anti-cytokeratin 19 antibody was performed on preoperative biopsy specimens, and patients with faint positive reactions were excluded. Intraoperatively, single-tracer SN biopsy with ICG fluorescence imaging was performed, followed by laparoscopic gastrectomy with modified D1+ or D2 lymph node dissection.

Results Twenty eligible patients underwent SN biopsy and laparoscopic gastrectomy. SNs were identified in 17 cases (85\%), with a median number of three SNs per patient. The median times for SN mapping and OSNA assay were 19 and 35 min, respectively. OSNA assay detected one metastatic lymph node, but all other nodes were negative. No adverse effects were observed in relation to $\mathrm{SN}$ mapping.

Conclusions Single-tracer SN mapping by ICG fluorescence imaging with intraoperative diagnosis by OSNA assay is feasible and safe. SNs can be identified in most patients, without producing false-negative results. Further clinical trial to demonstrate the sensitivity is ongoing.
\end{abstract}

Keywords Gastric cancer $\cdot$ Sentinel lymph node $\cdot$ Indocyanine green $\cdot$ Fluorescence imaging $\cdot$ Surgical pathology

Naoki Hiki

naoki.hiki@jfcr.or.jp

1 Department of Gastroenterological Surgery, Cancer Institute Hospital, Japanese Foundation for Cancer Research, 3-8-31 Ariake, Koto-ku, Tokyo 135-8550, Japan

2 Department of Gastroenterology, Cancer Institute Hospital, Japanese Foundation for Cancer Research, Tokyo, Japan

3 Department of Pathology, Cancer Institute Hospital, Japanese Foundation for Cancer Research, Tokyo, Japan

\section{Background}

Laparoscopic gastrectomy (LG) with lymphadenectomy is increasingly performed to manage early gastric cancer in Asian countries because of its reduced invasiveness compared with conventional open gastrectomy [1-6]. However, because the incidence of lymph node (LN) metastasis is low in early gastric cancer, LN dissection during LG may be overly invasive for patients with pathological N0 disease. Nevertheless, gastrectomy with D1+ or D2 lymphadenectomy has become a standard surgical procedure because the sensitivity of current preoperative imaging is low for detecting LNs.

To overcome the ambiguity of preoperative examinations for detecting $\mathrm{LN}$ metastasis, the sentinel node (SN) concept 
has been applied [7-14]. An SN is defined as the first draining $\mathrm{LN}$ that directly obtains lymphatic flow from the primary tumor [15]. If the $\mathrm{SN}$ is negative for cancer, it is posited that $\mathrm{LN}$ dissection may be omitted because metastasis to other LNs is unlikely. This concept has been applied clinically in surgery for melanoma and breast cancer $[15,16]$. However, a multicenter trial of SN biopsy for gastric cancer, using the dye-guided method with intraoperative injection of indocyanine green (ICG) [17], resulted in an unexpectedly high false-negative rate. These issues were probably technical, including the use of direct subserosal injection, the limited sensitivity of intraoperative histological diagnosis by hematoxylin and eosin staining for one section containing the largest LN dimension, and the learning period of only five patients per institution. Another prospective multicenter trial of SN mapping, performed by the endoscopic dualtracer method with radiolabeled tin colloid and blue dye [14], resulted in a favorable outcome when applied to clinical stage $\mathrm{T} 1$ gastric cancer $4 \mathrm{~cm}$ or less when the $\mathrm{SN}$ basin was resected. However, several barriers exist to the general application of current $\mathrm{SN}$ double-tracer biopsy methods for gastric cancer in clinical practice.

The first, and perhaps most important, issues relate to the invasiveness of the double-tracer method and the associated restrictions on the use of radioactive colloid. Because the dual-tracer method requires the injection of radiolabeled colloid on the day before surgery, patients must undergo additional endoscopic examination before the main surgery. Also, the use of radiolabeled colloid means that the dualtracer method can only be implemented in facilities with radiation-controlled endoscopic laboratories. Further, use of radioactive substances leads to high medical expenses [18, 19]. To overcome these limitations, intraoperative singletracer ICG fluorescence imaging has been introduced for SN mapping during gastric cancer surgery, and to date, has produced favorable outcomes [20-25]. SN biopsy by ICG fluorescence during LG has been facilitated by the development of laparoscopic ICG fluorescence imaging systems $[24,25]$.

The second major issue relates to the inspection accuracy and restriction of intraoperative pathological diagnosis of metastasis in SNs. In multi-institutional research, intraoperative diagnosis was not available in $24 \%$ of patients because of the pathology policies in institutions [14]. The reported sensitivities of intraoperative histological diagnosis that relied on hematoxylin and eosin staining of a representative cut surface of a frozen section were $79 \%$ and $70 \%$ when based on patients and LNs, respectively [14]. This suggested the need for more accurate examination. One-step nucleic acid amplification (OSNA) assay, which directly amplifies the mRNA of the molecular marker cytokeratin 19 (CK19) from the supernatant of homogenized LNs, has been shown to be able to diagnose $\mathrm{LN}$ metastasis within $30 \mathrm{~min}$, and is already in clinical use in surgery for breast and colorectal cancer in Japan [26-28]. Recent studies have confirmed the clinical utility of OSNA assay for detecting LN metastasis in gastric cancer [26, 29].

In this study, we aimed to demonstrate the feasibility and safety of SN mapping by single-tracer ICG fluorescence imaging, with the intraoperative diagnosis of $\mathrm{LN}$ metastasis by OSNA assay, during LG for gastric cancer. This approach may permit function-preserving, minimally invasive gastrectomy without the need for lymphadenectomy in patients with early gastric cancer who have no LN metastasis.

\section{Methods}

\section{Study design}

This was a single-arm, prospective study to verify the feasibility of SN mapping by ICG fluorescence imaging and of metastatic diagnosis by OSNA during LG among patients with early gastric cancer. The primary endpoint was the detection rate of fluorescent LNs. The secondary endpoints were as follows: the times for ICG injection, SN biopsy, and OSNA assay; the number and distribution of fluorescent LNs; and the adverse effects that occurred. Because this was a feasibility study, the sample size was limited to 20 patients. All patients provided written informed consent. The study protocol was approved by the Ethics Committee of the Cancer Institute Hospital of JFCR (approval number: 2016-1063) and the study was conducted in accordance with the principles of the Declaration of Helsinki.

\section{Patients}

Patients with histologically confirmed clinical T1NOM0 gastric adenocarcinoma preoperatively, for whom the single primary lesion measured $4 \mathrm{~cm}$ or less and had not been treated (including endoscopically), were considered for inclusion. For each patient, surgery and ICG injection were performed or supervised by experts in laparoscopic surgery and endoscopic treatment. The tumor depth and extent of nodal involvement were determined by preoperative evaluations, including barium radiography, upper gastrointestinal tract endoscopy, and computed tomography, and were evaluated using the "Japanese classification of gastric carcinoma: 3rd English edition" [30]. Additional preoperative endoscopic ultrasonography was performed in the patients with tumor whose invasion depth was difficult to assess by endoscopic examination.

According the Japanese gastric cancer treatment guidelines 2014, version 4, [31], patients who met the criteria for endoscopic resection underwent endoscopic submucosal dissection and were excluded. Patients with a history 
of drug-related allergy or active asthma were also excluded because of the potential risk of anaphylactic reaction to ICG. Immunohistochemical staining was performed with the anti-CK19 antibody on preoperative biopsy specimens, and patients with faint positive reactions were excluded because a low CK19 expression could have produced false-negative test results [29]. Additionally, to avoid inaccuracy due to random distributions of metastatic deposits within an LN [29], OSNA assay was performed for the whole SN. Patients with apparent T2, T3, or T4 tumors, or with extensive abdominal adhesions, during surgery were also excluded.

\section{Intraoperative SN mapping and OSNA assay}

The surgical procedure was performed under a pneumoperitoneum created by injecting carbon dioxide at $10-12 \mathrm{mmHg}$, before inserting five ports $(5-12 \mathrm{~mm})$. The ICG, endoscopic puncture needle, and laboratory consumables used for the OSNA assay were donated by Sysmex Corp. (Kobe, Japan). The greater omentum was dissected before ICG injection to observe the suprapancreatic lymph flow. ICG (Diagnogreen, Dai-Ichi Sankyo Pharm. Co., Tokyo, Japan), diluted 100 times in a total volume of $2 \mathrm{~mL}$, was injected in four divisions around the primary tumor using an intraoperative endoscopic puncture needle. Intraperitoneal observation was performed for $20 \mathrm{~min}$ at most, using a laparoscope and an ICG fluorescence imaging system (K26003 BGA HOPKINS ${ }^{\circledR}$ ICG telescope, KARL STORZ SE \& Co., Tuttlingen, Germany, or VISERA ELITE II telescope IR, OLYMPUS Co., Tokyo, Japan) immediately following ICG injection (Fig. 1). A maximum of four fluorescent $\mathrm{LNs}$ were selected and excised before gastrectomy. When more than four fluorescent LNs were detected, the four with the highest fluorescence intensities were selected due to the mechanical restrictions of the RD-100i system (Sysmex).

Diagnosis of metastasis in an SN was performed by OSNA assay immediately after its removal, as previously described [29]. Briefly, the whole SN was homogenized using a LYNORHAG lysis buffer (Sysmex) and centrifuged at $10,000 \times g$. Then, the LYNOAMP BC gene amplification reagent (Sysmex) was added to the supernatant, and the CK19 mRNA content in each lysate was amplified and detected with the RD-100i system (Sysmex). Amplification times were analyzed based on a previously generated standard curve, and the assay results were expressed as the number of CK19 mRNA copies per microliter. The cutoff value for positive LNs was set at 250 copies/ $\mu \mathrm{L}$, based on previous research [29, 32]. Non-fluorescent LNs underwent histological examination by HE staining of one representative cut surface of a paraffin-embedded specimen.

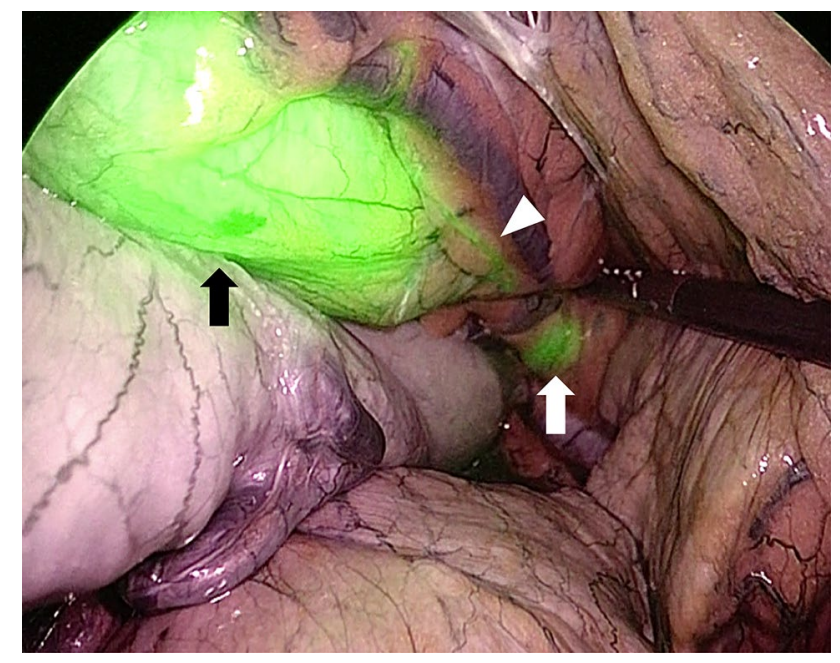

Fig. 1 Observation of sentinel nodes by indocyanine green (ICG) fluorescence imaging. Intraperitoneal observation was performed immediately after ICG injection. Fluorescence of lymphatic vessels (arrowhead) and lymph nodes (white arrow) was done in real time. The black arrow indicates the endoscopically injected ICG

\section{Gastrectomy and LN dissection}

After SN mapping, patients underwent laparoscopic distal gastrectomy (LDG) or laparoscopic pylorus-preserving gastrectomy (LPPG) with modified D1+ (D1+8 a, 9, 11p) or D2 lymphadenectomy, as previously reported [33-35]. The surgical procedure was selected by tumor location: LPPG was performed in patients with tumors in the middle-third of the stomach that were at least $5 \mathrm{~cm}$ from the pyloric ring with no evidence of regional LN metastasis, while LDG was performed in all other patients. After gastrectomy, intracorporeal gastro-gastro anastomosis was performed in cases that underwent LPPG [36], and an intracorporeal Billroth-I [37] or a Roux-en-Y [33] reconstruction was performed in cases that underwent LDG.

\section{Data collection}

Patient data were collected, including the following: age, sex, height, weight, and body mass index; medical history; tumor characteristics, such as location, preoperative size, and clinical $\mathrm{T}$ and $\mathrm{N}$ stages; surgical variables such as surgical procedure, operation time, intraoperative blood loss, and extent of lymphadenectomy; postoperative courses such as the presence or absence of complications and hospital stays; and pathological results such as the number of retrieved LNs, the tumor size, the pathological $\mathrm{T}$ and $\mathrm{N}$ stage, and the histological type. We also collected the following SN mapping and OSNA assay details: the times for ICG injection, SN biopsy, and OSNA assay; the number and location of SNs; and the results of the OSNA assay. In patients with 
complications, the details and the relevance of those complications to the SN mapping procedure were investigated. Unless otherwise stated, data are presented as medians and range.

\section{Results}

\section{Patients and surgical treatment}

From April 2017 to January 2018, 33 patients were preoperatively considered for inclusion in this study. Four patients (12\%) were excluded from the study by faint positive immunohistochemical staining results, and seven patients were excluded preoperatively by other patient factors. Two patients were excluded intraoperatively because of severe intra-abdominal adhesions and an apparent T4 tumor. Ultimately, 20 patients underwent SN biopsy and subsequent LG (Table 1), with LPPG and LDG used in 11 and 9 patients, respectively. Two patients underwent $\mathrm{D} 2 \mathrm{LN}$ dissection: one because of suspected muscular invasion in the surgical

Table 1 Preoperative and operative variables

\begin{tabular}{|c|c|}
\hline Age (years) & $61(38-80)$ \\
\hline Male/female & $6 / 14$ \\
\hline Height (cm) & $157.0(147.9-180.1)$ \\
\hline Weight $(\mathrm{kg})$ & $53.8(41.8-76.9)$ \\
\hline Body mass index $\left(\mathrm{kg} / \mathrm{m}^{2}\right)$ & $21.7(17.2-25.0)$ \\
\hline Location: $\mathrm{U}^{\mathrm{a}} / \mathrm{M}^{\mathrm{b}} / \mathrm{L}^{\mathrm{c}}$ & $1 / 13 / 6$ \\
\hline $\begin{array}{l}\text { Cross-sectional circumference: Ant }{ }^{\mathrm{d}} / \mathrm{Post}^{\mathrm{e}} / \mathrm{Gre}^{\mathrm{f}} / \\
\text { Less }^{\mathrm{g}}\end{array}$ & $3 / 6 / 5 / 6$ \\
\hline Size of the primary tumor (mm) & $25(15-40)$ \\
\hline Biopsy: differentiated/undifferentiated & $6 / 14$ \\
\hline Tumor depth: cT1a/cT1b & $6 / 14$ \\
\hline Surgical procedure: $\mathrm{LPPG}^{\mathrm{h}} / \mathrm{LDG}^{\mathrm{i}}$ & $11 / 9$ \\
\hline Field of lymph-node dissection: D1+/D2 & $18 / 2$ \\
\hline $\begin{array}{l}\text { Reconstruction after LDG: Billroth-I/Roux- } \\
\text { en-Y }\end{array}$ & $5 / 4$ \\
\hline Operative duration (min) & $291(229-387)$ \\
\hline Intraoperative blood loss $(\mathrm{mL})$ & $13(5-110)$ \\
\hline Postoperative hospital stay (days) & $10(9-24)$ \\
\hline Complication & $3(15 \%)$ \\
\hline
\end{tabular}

${ }^{\mathrm{a}}$ Upper-third of the stomach

${ }^{b}$ Middle-third of the stomach

${ }^{c}$ Lower-third of the stomach

${ }^{\mathrm{d}}$ Anterior wall side

${ }^{\text {e}}$ Posterior wall side

${ }^{\mathrm{f}}$ Greater curvature side

${ }^{\mathrm{g}}$ Lesser curvature side

${ }^{\mathrm{h}}$ Laparoscopic pylorus-preserving gastrectomy

${ }^{\mathrm{i}}$ Laparoscopic distal gastrectomy specimen, and the other because of the SN biopsy result (described below). Modified D1+ LN dissection was performed for all other patients.

Three patients (15\%) suffered postoperative complications, which included surgical site infection, delayed gastric emptying, and pneumonia. All patients with complications were treated conservatively. No adverse events occurred in relation to either the ICG injection or the SN mapping. The median postoperative hospital stay after SN mapping and LG was 10 days (Table 1).

\section{Results of SN biopsy and OSNA assay}

The detection rate of SNs by ICG fluorescence imaging was $85 \%$ (17/20) (Table 2). After gastrectomy, fluorescent LNs were identified in all resected specimens, including the three cases in which SNs were undetected during operation. The SN distributions, based on the cross-sectional location of the primary tumors, are shown in Fig. 2. As shown, SNs were usually perigastric, although we also observed one along the left gastric artery, one in a suprapancreatic site, and three along the common hepatic artery.

The median number of SNs was three, and the median total time for SN mapping was 19 min in the detected cases. Overall, 48 SNs were subject to OSNA assay intraoperatively, taking a median time of 35 min per patient. One SN retrieved from a patient with a $25-\mathrm{mm}$ clinical stage $\mathrm{T} 1 \mathrm{~b}$ tumor of the anterior wall of the middle gastric body, who was scheduled for LPPG, was found to be positive (station no. 3a, with 460 copies $/ \mu \mathrm{L}$ ) and was converted to LDG with D2 LN dissection. All other LNs were negative on the OSNA

Table 2 Results of sentinel lymph-node mapping and histological examination

\begin{tabular}{ll}
\hline Time for ICG ${ }^{\mathrm{a}}$ injection (min) & $2(1-4)$ \\
Observation time (min) & $4(1-20)$ \\
$\mathrm{SN}^{\mathrm{b}}$ identified cases & $17(85 \%)$ \\
Time for SN biopsy (min) & $10(3-25)$ \\
Total time for SN mapping (min) & $19(11-33)$ \\
Time for OSNA ${ }^{\mathrm{c}}$ assay (min) & $35(25-48)$ \\
Number of SN & $3(0-4)$ \\
Positive SN & 1 \\
Size of the primary tumor, mm & $27(12-60)$ \\
Histological type: differentiated/undifferentiated & $6 / 14$ \\
Tumor depth: pT1a/pT1b/pT2 & $12 / 6 / 2$ \\
Nodal involvement: N0/N1 & $19 / 1$ \\
Retrieved lymph nodes & $38(24-62)$ \\
\hline${ }^{\mathrm{a}}$ Indocyanine green & \\
${ }^{\mathrm{b}}$ Sentinel node & \\
${ }^{\mathrm{c}}$ One-step nucleic acid amplification
\end{tabular}


Fig. 2 Sentinel node (SN) sites at each cross-sectional circumference. a Anterior wall, b greater curvature, $\mathbf{c}$ lesser curvature, and $\mathbf{d}$ posterior wall. Red and blue circles indicate the primary tumor and $\mathrm{SN}$ locations, respectively. Arabic numbers indicate the lymph node station according to the Japanese classification of gastric carcinoma [30]. Most SNs were located at perigastric sites, but were also observed at suprapancreatic sites, along the left gastric artery, and along the common hepatic artery
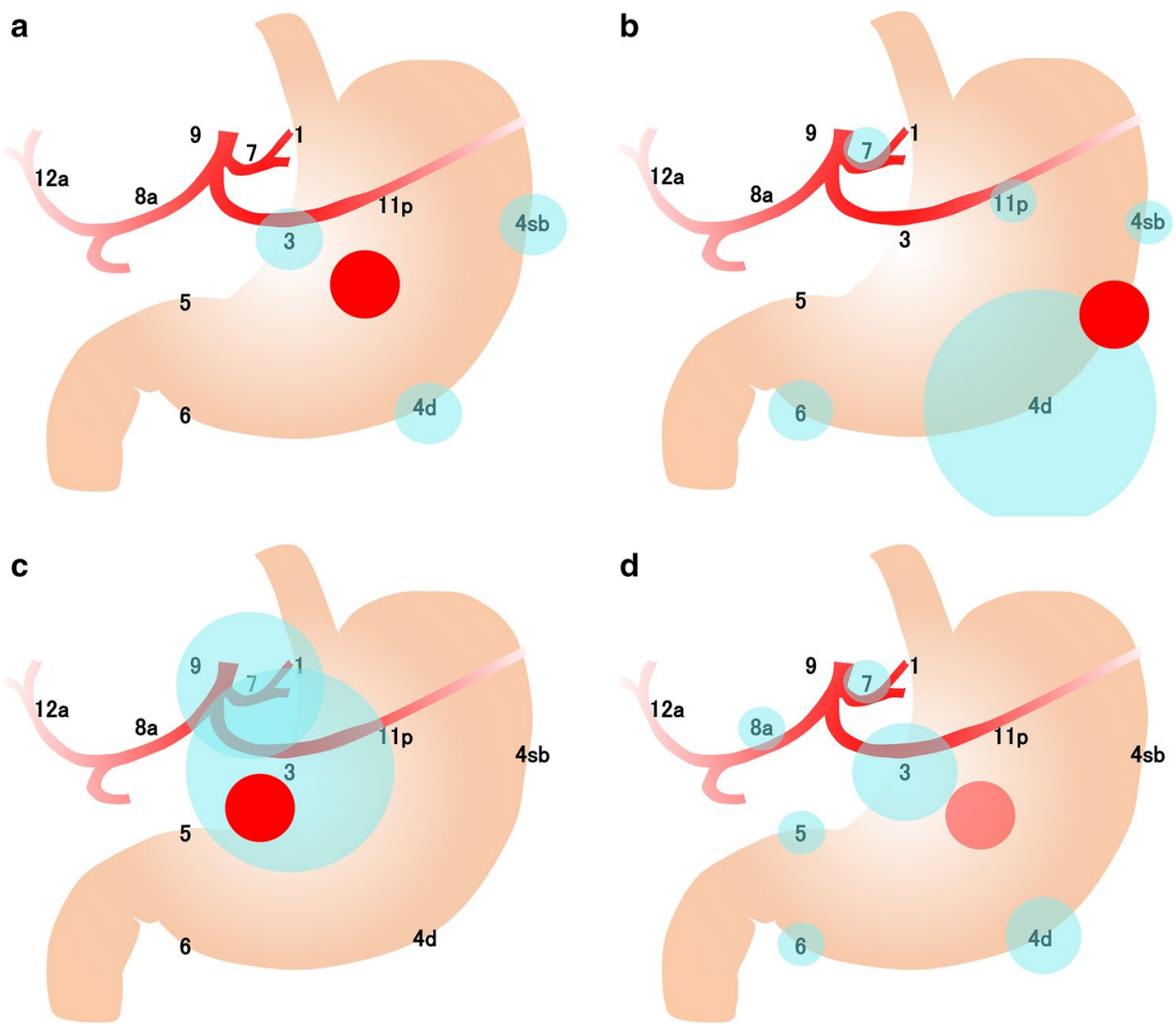

assay. Histological examination of the $721 \mathrm{LNs}$ retrieved from all patients detected no positive LNs (Table 2).

\section{Diagnostic accuracy of the endoscopic examination for the primary tumor}

Regarding tumor depth, 19 tumors $(95 \%)$ were diagnosed as pathological T1; however, one tumor that was diagnosed by preoperative endoscopic examination as clinical $\mathrm{T} 1 \mathrm{~b}$ was diagnosed by histological examination as pathological $\mathrm{T} 2$. Regarding tumor diameter, 18 (90\%) of the tumors measured $4 \mathrm{~cm}$ or less by histological examination, but two tumors measuring 4 and $3 \mathrm{~cm}$ on preoperative endoscopic examination actually measured 6 and $4.5 \mathrm{~cm}$ on histological examination, respectively.

\section{Discussion}

In this prospective study, we sought to demonstrate the feasibility and safety of single-tracer SN mapping by ICG fluorescence imaging with metastatic diagnosis by OSNA assay. The procedures were performed successfully and rapidly, with no false-negative cases or adverse effects. However, the $\mathrm{SN}$ detection rate was $85 \%$, which was relatively low when compared with previous reports [14, 39].
SNs were not identified in three cases (the first, fourth, and seventeenth cases), suggesting that the learning curve for the procedure may not be the reason for non-detection. Additionally, fluorescent LNs were observed in the resected specimens of all 20 cases, including the three cases that were not identifiable during the intraoperative observation period, which would indicate that the ICG injection was performed properly in all cases. Further, fluorescent LNs in the resected specimen for those three cases were located at the perigastric site, suggesting that the loss of visibility in adipose tissues may also not be the reason for non-detection. The difference in lymph flow velocity among patients could account for the failure to detect SNs in these three cases because the observation period was limited to just $20 \mathrm{~min}$ after endoscopic tracer injection. Longer observation times might have been required for those patients. However, because intraoperative injection was chosen in this study in order to observe the lymphatic flow in real time and to lessen the invasiveness of endoscopic examination, 20 min may be a realistic observation period. Patients in whom SNs are not identified will undergo conventional gastrectomy with $\mathrm{LN}$ dissection. Therefore, low detection rate should not result in an unfavorable oncological outcome. Detection rate of SNs would be reassessed in the ongoing clinical trial.

In this study, most SNs were located at perigastric sites, but four were identified at LN stations 7, 8a, and 11p. This 
is comparable to the $\mathrm{SN}$ distribution reported in a previous single-center study [7]. Of note, those latter SNs are typically difficult to recognize by dye-guided methods because visibility is lost in adipose tissues [25], but the use of ICG fluorescence imaging overcame this limitation. Additionally, the single-tracer method with endoscopic ICG injection performed intraoperatively made it possible to omit additional endoscopic injection of radiolabeled colloid preoperatively, as is needed for the conventional dual-tracer method [14, 38].

Intraoperative diagnosis of metastatic SNs by OSNA assay was performed within $48 \mathrm{~min}$ in each patient, and this approach did not produce any false-negative cases. One patient with a positive SN may also have benefited from rapid inspection, because surgery was converted to a D2 LN dissection, without the need for additional treatment postoperatively. OSNA assay was limited to four LNs per patient due to mechanical restrictions in the study, but most patients had three or fewer fluorescent LNs, suggesting that this mechanical restriction was unlikely to have affected the result. Additionally, the new gene amplification detector RD-200 (Sysmex) increases the maximum number of samples that can be measured simultaneously to 14 samples. Use of the new detector may withdraw the restriction.

There were some important limitations to this study that should be addressed. Among the 20 patients who were included, only one had a tumor in the upper-third of the stomach, with the remaining 19 having tumors of the middle or lower two-thirds of the stomach. Therefore, feasibility and safety of SN mapping by ICG fluorescence for upperthird gastric cancer remain unclear. Patients with upper-third gastric cancer may gain the greatest benefit from SN biopsy by being able to avoid total gastrectomy and undergo function-preserving gastrectomy instead. Further accumulation of cases is necessary in future clinical trials to verify the feasibility and efficacy of SN mapping by ICG fluorescence imaging, especially for upper-third early gastric cancer.

Additionally, patients were excluded from this study if they had previously undergone endoscopic resection. However, studies have shown that the SN is not significantly affected by endoscopic resection [39, 40]. After non-curative endoscopic resection, patients may therefore benefit from SN biopsy, because further gastrectomy and LN dissection may be omitted if SNs are negative for metastasis.

Finally, the application of SN mapping was limited to patients with clinical T1N0 gastric cancer, based on the results of a multicenter trial in Japan [14]. This may also be problematic because LN metastasis rarely occurs in this population [41]. We also limited the target population to 20 in this study because we only aimed to assess the feasibility and safety of the single-tracer method. Consequently, LN metastasis was only observed in one patient during the study, calling into question the method's accuracy and whether SN basin resection is necessary. A clinical trial to resolve these issues is needed. The following trial is a single-arm, prospective study to evaluate the accuracy of SN mapping by ICG fluorescence imaging and intraoperative diagnosis of LN metastasis by OSNA assay. The primary endpoint of the study is false-negative rate. We calculated that 20 patients with LN metastasis are needed to test the null hypothesis for false-negative rate which is not more than $15 \%$ when onesided alpha $=0.05$ and power for the alternative hypothesis which is $1 \%$ is more than $80 \%$ by the exact binomial test. Assuming that the rate of $\mathrm{LN}$ metastasis in this population is $10-20 \%$, the sample size is set at 150 . This clinical trial is now ongoing.

\section{Conclusions}

SN mapping by ICG fluorescence imaging and intraoperative diagnosis of LN metastasis by OSNA assay are safe and feasible. SNs were identified in most patients and there were no false-negative cases or complications related to $\mathrm{SN}$ mapping. A clinical trial into the accuracy of the method is ongoing.

Acknowledgements The authors would like to thank Yuka Toyama, Hiroko Nagano, Naoko Takahashi, and Erina Okada (Department of Pathology, Cancer Institute Hospital, Japanese Foundation for Cancer Research) for providing technical assistance with the OSNA assay.

\section{Compliance with ethical standards}

Conflict of interest The authors declare that they have no conflict of interest.

Ethical standards All procedures followed were in accordance with the ethical standards of the responsible committee on human experimentation (institutional and national) and with the Helsinki Declaration of 1964 and later versions. Informed consent to be included in the study, or the equivalent, was obtained from all patients.

\section{References}

1. Katai H, Sasako M, Fukuda H, Nakamura K, Hiki N, Saka M, et al. Safety and feasibility of laparoscopy-assisted distal gastrectomy with suprapancreatic nodal dissection for clinical stage I gastric cancer: a multicenter phase II trial (JCOG 0703). Gastric Cancer. 2010;13(4):238-44.

2. Kitano S, Shiraishi N, Uyama I, Sugihara K, Tanigawa N, Japanese Laparoscopic Surgery Study G. A multicenter study on oncologic outcome of laparoscopic gastrectomy for early cancer in Japan. Ann Surg. 2007;245(1):68-72.

3. Kitano S, Shiraishi N. Current status of laparoscopic gastrectomy for cancer in Japan. Surg Endosc. 2004;18(2):182-5.

4. Zeng YK, Yang ZL, Peng JS, Lin HS, Cai L. Laparoscopy-assisted versus open distal gastrectomy for early gastric cancer: evidence from randomized and nonrandomized clinical trials. Ann Surg. 2012;256(1):39-52. 
5. Hiki N, Katai H, Mizusawa J, Nakamura K, Nakamori M, Yoshikawa T, et al. Long-term outcomes of laparoscopy-assisted distal gastrectomy with suprapancreatic nodal dissection for clinical stage I gastric cancer: a multicenter phase II trial (JCOG0703). Gastric Cancer. 2017;21(1):155-61.

6. Kim W, Kim HH, Han SU, Kim MC, Hyung WJ, Ryu SW, et al. Decreased morbidity of laparoscopic distal gastrectomy compared with open distal gastrectomy for stage I gastric cancer: shortterm outcomes from a multicenter randomized controlled trial (KLASS-01). Ann Surg. 2016;263(1):28-35.

7. Takeuchi H, Goto O, Yahagi N, Kitagawa Y. Function-preserving gastrectomy based on the sentinel node concept in early gastric cancer. Gastric Cancer. 2017;20(Suppl 1):53-9.

8. Hiratsuka M, Miyashiro I, Ishikawa O, Furukawa $\mathrm{H}$, Motomura K, Ohigashi $\mathrm{H}$, et al. Application of sentinel node biopsy to gastric cancer surgery. Surgery. 2001;129(3):335-40.

9. Kitagawa Y, Fujii H, Mukai M, Kubota T, Otani Y, Kitajima M. Radio-guided sentinel node detection for gastric cancer. Br J Surg. 2002;89(5):604-8.

10. Miwa K, Kinami S, Taniguchi K, Fushida S, Fujimura T, Nonomura A. Mapping sentinel nodes in patients with early-stage gastric carcinoma. Br J Surg. 2003;90(2):178-82.

11. Hayashi H, Ochiai T, Mori M, Karube T, Suzuki T, Gunji Y, et al. Sentinel lymph node mapping for gastric cancer using a dual procedure with dye- and gamma probe-guided techniques. J Am Coll Surg. 2003;196(1):68-74.

12. Isozaki H, Kimura T, Tanaka N, Satoh K, Matsumoto S, Ninomiya M, et al. An assessment of the feasibility of sentinel lymph node-guided surgery for gastric cancer. Gastric Cancer. 2004;7(3):149-53.

13. Arigami T, Natsugoe S, Uenosono Y, Mataki Y, Ehi K, Higashi H, et al. Evaluation of sentinel node concept in gastric cancer based on lymph node micrometastasis determined by reverse transcription-polymerase chain reaction. Ann Surg. 2006;243(3):341-7.

14. Kitagawa Y, Takeuchi H, Takagi Y, Natsugoe S, Terashima M, Murakami N, et al. Sentinel node mapping for gastric cancer: a prospective multicenter trial in Japan. J Clin Oncol. 2013;31(29):3704-10.

15. Morton DL, Wen DR, Wong JH, Economou JS, Cagle LA, Storm FK, et al. Technical details of intraoperative lymphatic mapping for early stage melanoma. Arch Surg. 1992;127(4):392-9.

16. Giuliano AE, Kirgan DM, Guenther JM, Morton DL. Lymphatic mapping and sentinel lymphadenectomy for breast cancer. Ann Surg. 1994;220(3):391-8 (discussion 8-401).

17. Miyashiro I, Hiratsuka M, Sasako M, Sano T, Mizusawa J, Nakamura K, et al. High false-negative proportion of intraoperative histological examination as a serious problem for clinical application of sentinel node biopsy for early gastric cancer: final results of the Japan Clinical Oncology Group multicenter trial JCOG0302. Gastric Cancer. 2014;17(2):316-23.

18. Bostick P, Essner R, Glass E, Kelley M, Sarantou T, Foshag LJ, et al. Comparison of blue dye and probe-assisted intraoperative lymphatic mapping in melanoma to identify sentinel nodes in 100 lymphatic basins. Arch Surg. 1999;134(1):43-9.

19. Giuliano AE, Jones RC, Brennan M, Statman R. Sentinel lymphadenectomy in breast cancer. J Clin Oncol. 1997;15(6):2345-50.

20. Nimura H, Narimiya N, Mitsumori N, Yamazaki Y, Yanaga K, Urashima M. Infrared ray electronic endoscopy combined with indocyanine green injection for detection of sentinel nodes of patients with gastric cancer. Br J Surg. 2004;91(5):575-9.

21. Ishikawa K, Yasuda K, Shiromizu A, Etoh T, Shiraishi N, Kitano S. Laparoscopic sentinel node navigation achieved by infrared ray electronic endoscopy system in patients with gastric cancer. Surg Endosc. 2007;21(7):1131-4.

22. Kusano M, Tajima Y, Yamazaki K, Kato M, Watanabe M, Miwa M. Sentinel node mapping guided by indocyanine green fluorescence imaging: a new method for sentinel node navigation surgery in gastrointestinal cancer. Dig Surg. 2008;25(2):103-8.

23. Tajima Y, Yamazaki K, Masuda Y, Kato M, Yasuda D, Aoki T, et al. Sentinel node mapping guided by indocyanine green fluorescence imaging in gastric cancer. Ann Surg. 2009;249(1):58-62.

24. Tajima Y, Murakami M, Yamazaki K, Masuda Y, Kato M, Sato A, et al. Sentinel node mapping guided by indocyanine green fluorescence imaging during laparoscopic surgery in gastric cancer. Ann Surg Oncol. 2010;17(7):1787-93.

25. Miyashiro I, Kishi K, Yano M, Tanaka K, Motoori M, Ohue M, et al. Laparoscopic detection of sentinel node in gastric cancer surgery by indocyanine green fluorescence imaging. Surg Endosc. 2011;25(5):1672-6.

26. Yamamoto H, Sekimoto M, Oya M, Yamamoto N, Konishi F, Sasaki J, et al. OSNA-based novel molecular testing for lymph node metastases in colorectal cancer patients: results from a multicenter clinical performance study in Japan. Ann Surg Oncol. 2011;18(7):1891-8.

27. Tamaki Y, Akiyama F, Iwase T, Kaneko T, Tsuda H, Sato K, et al. Molecular detection of lymph node metastases in breast cancer patients: results of a multicenter trial using the one-step nucleic acid amplification assay. Clin Cancer Res. 2009;15(8):2879-84.

28. Godey F, Leveque J, Tas P, Gandon G, Poree P, Mesbah H, et al. Sentinel lymph node analysis in breast cancer: contribution of one-step nucleic acid amplification (OSNA). Breast Cancer Res Treat. 2012;131(2):509-16.

29. Kumagai K, Yamamoto N, Miyashiro I, Tomita Y, Katai H, Kushima R, et al. Multicenter study evaluating the clinical performance of the OSNA assay for the molecular detection of lymph node metastases in gastric cancer patients. Gastric Cancer. 2014;17(2):273-80.

30. Japanese Gastric Cancer A. Japanese classification of gastric carcinoma: 3rd English edition. Gastric Cancer. 2011;14(2):101-12.

31. Japanese Gastric Cancer A. Japanese gastric cancer treatment guidelines 2014 (ver. 4). Gastric Cancer. 2017;20(1):1-19.

32. Yaguchi $Y$, Sugasawa $H$, Tsujimoto $H$, Takata $H$, Nakabayashi $K$, Ichikura T, et al. One-step nucleic acid amplification (OSNA) for the application of sentinel node concept in gastric cancer. Ann Surg Oncol. 2011;18(8):2289-96.

33. Kumagai K, Hiki N, Nunobe S, Jiang X, Kubota T, Aikou S, et al. Different features of complications with Billroth-I and Roux-en$\mathrm{Y}$ reconstruction after laparoscopy-assisted distal gastrectomy. J Gastrointest Surg. 2011;15(12):2145-52.

34. Tsujiura M, Hiki N, Ohashi M, Nunobe S, Kumagai K, Ida S, et al. Excellent long-term prognosis and favorable postoperative nutritional status after laparoscopic pylorus-preserving gastrectomy. Ann Surg Oncol. 2017;24(8):2233-40.

35. Kumagai K, Hiki N, Nunobe S, Kamiya S, Tsujiura M, Ida S, et al. Impact of anatomical position of the pancreas on postoperative complications and drain amylase concentrations after laparoscopic distal gastrectomy for gastric cancer. Surg Endosc. 2018;32(9):3846-54.

36. Kumagai K, Hiki N, Nunobe S, Sekikawa S, Chiba T, Kiyokawa $\mathrm{T}$, et al. Totally laparoscopic pylorus-preserving gastrectomy for early gastric cancer in the middle stomach: technical report and surgical outcomes. Gastric Cancer. 2015;18(1):183-7.

37. Kanaya S, Kawamura Y, Kawada H, Iwasaki H, Gomi T, Satoh $\mathrm{S}$, et al. The delta-shaped anastomosis in laparoscopic distal gastrectomy: analysis of the initial 100 consecutive procedures of intracorporeal gastroduodenostomy. Gastric Cancer. 2011;14(4):365-71.

38. Kitagawa Y, Fujii H, Mukai M, Kubota T, Ando N, Watanabe $\mathrm{M}$, et al. The role of the sentinel lymph node in gastrointestinal cancer. Surg Clin N Am. 2000;80(6):1799-809.

39. Mayanagi S, Takeuchi H, Kamiya S, Niihara M, Nakamura R, Takahashi T, et al. Suitability of sentinel node mapping as an 
index of metastasis in early gastric cancer following endoscopic resection. Ann Surg Oncol. 2014;21(9):2987-93.

40. Arigami T, Uenosono Y, Yanagita S, Matsushita D, Arima H, Hirata M, et al. Feasibility of sentinel node navigation surgery after noncurative endoscopic resection for early gastric cancer. $\mathbf{J}$ Gastroenterol Hepatol. 2013;28(8):1343-7.

41. Sekiguchi M, Oda I, Taniguchi H, Suzuki H, Morita S, Fukagawa $\mathrm{T}$, et al. Risk stratification and predictive risk-scoring model for lymph node metastasis in early gastric cancer. J Gastroenterol. 2016;51(10):961-70.

Publisher's Note Springer Nature remains neutral with regard to jurisdictional claims in published maps and institutional affiliations. 